\title{
What drives 20th century polar motion?
}

\author{
Surendra Adhikari ${ }^{a, *}$, Lambert Caron ${ }^{\mathrm{a}}$, Bernhard Steinberger ${ }^{\mathrm{b}, \mathrm{c}}$, John T. \\ Reager $^{\mathrm{a}}$, Kristian K. Kjeldsen ${ }^{\mathrm{d}, \mathrm{e}}$, Ben Marzeion $^{\mathrm{f}}$, Eric Larour ${ }^{\mathrm{a}}$, Erik R. Ivins ${ }^{\mathrm{a}}$ \\ ${ }^{a}$ Jet Propulsion Laboratory, California Institute of Technology, Pasadena, California, USA \\ ${ }^{b}$ GFZ German Research Centre for Geosciences, Potsdam, Germany. \\ ${ }^{c}$ Centre for Earth Evolution and Dynamics, University of Oslo, Oslo, Norway. \\ ${ }^{d}$ Department of Geodesy, Technical University of Denmark, Kongens Lyngby, Denmark. \\ ${ }^{e}$ Geological Survey of Denmark and Greenland, Copenhagen, Denmark. \\ ${ }^{f}$ Institute of Geography, University of Bremen, Bremen, Germany.
}

\begin{abstract}
Astrometric and geodetic measurements show that the mean position of Earth's spin axis drifted through the solid crust toward Labrador, Canada at an average speed of $10.5 \pm 0.9 \mathrm{~cm} /$ year during the 20 th century. Understanding the origins of this secular polar motion (SPM) has significance for modeling the global climate, as it provides a link to ice mass balance and sea-level rise. A perplexing issue, however, is that while glacial isostatic adjustment (GIA) models satisfactorily explain the direction of SPM, the associated prediction of the amplitude is insufficient. Our Bayesian GIA analysis, with constraints from relative sea-level and vertical land motion data, reveals that this process only accounts for $33 \pm 18 \%$ of the observed SPM amplitude. This shortfall motivates a more broadly scoped reassessment of SPM drivers. To address this, we assemble a complete reconstruction of Earth's surface mass transport derived from recent advancements in modeling the global 20th century cryospheric, hydrologic, oceanic, and seismogenic mass exchange. The summed signals, nonetheless, cannot fully reconcile the observed SPM, even when considering the error statistics of each driver. We investigate an additional excitation source: changes in Earth's inertia tensor caused by mantle convection. Sophisticated models
\end{abstract}

\footnotetext{
* Corresponding author

Email address: surendra.adhikari@jpl.nasa.gov (Surendra Adhikari)

URL: https://science.jpl.nasa.gov/people/Adhikari/ (Surendra Adhikari)
} 
have recently been advanced in tectonic plate reconstructions, in conjunction with geoid and seismic tomographic models. Here we use these models to compute new estimates of SPM. While the convection-driven SPM has considerable uncertainty, the average direction of 283 recent models aligns with the residual SPM (within $2.7^{\circ} \pm 14.8^{\circ}$ ), significantly reducing the gap between observation and prediction. We assert that one key mechanism for driving 20th century SPM is long-term mass movement due to mantle convection.

Keywords: Earth rotation, polar motion, glacial isostatic adjustment, surface mass transport, mantle convection

\section{Introduction}

The study of the movement of Earth's spin axis through the Earth's crust in the astrometric and space geodetic observing era (1899-present) may be divided into at least four elements that differ by their respective timescales: hours 5 to weeks generally involve tides, winds and atmospheric/oceanic forcings; annual and 433-day Chandler periods involve global solar related forcing and a free wobble, respectively; interannual, interdecadal and 30-year Markowitz periods involve global hydrological and cryospheric forcings, possibly modified by a subtle core-mantle coupling. The subject of this paper involves the remain-

10 ing timescale of observation: a secular movement of the spin axis since 1899 . Combining all available estimates [1] suggests that the spin axis drifted along $74.2^{\circ} \pm 4.7^{\circ}$ west longitude at a speed of $10.5 \pm 0.9 \mathrm{~cm} /$ year during the $20 \mathrm{th}$ century (Figure 1a). The basic theoretical relationship of Earth's surface and interior mass transport and changes to the inertia tensor, and hence polar motion, is well known [2, 3, 4, 5, 6, 7]. The question, however, is the following: which aspects of mass transport are dominant drivers of the 20th century SPM?

Here we analyze two of the Earth's interior viscous mass transport processes that have much longer timescales than the polar motion observations themselves: GIA and mantle convection that operate on timescales of thousands to tens of millions of years. In addition, we comprehensively account for contemporary 
environmental forcings that involve global surface mass transport (e.g., glaciers and ice sheets imbalances, sea-level change) and the net effects of seismic deformation, in order to deliver a new, multidisciplinary, and unified explanation to the 20th century polar motion.

\section{2. Glacial isostatic adjustment}

It has been argued throughout the last four decades that slow viscous mantle flow in response to many cycles of Late Pleistocene glaciation drives the observed SPM [2, 3, 6]. For a reasonable choice of deglaciation history, solid Earth structure, and material parameters (especially lower mantle viscosity),

so it is indeed possible to construct a GIA model that matches both the direction and amplitude of observed SPM almost entirely (Figure 1a). This simple explanation, however, is highly problematic because it ignores the changes in Earth's inertia tensor accompanying an unequivocal rise in global mean sealevel (GMSL) during the 20th century [8, 9]. One recent breakthrough in our

35 understanding of GIA processes, for example, is the recognition of an important restoring torque due to the background long-term triaxiality of the Earth's inertia tensor [7. Such necessary improvements in the GIA model generally dampen the predicted SPM amplitudes (Figure 1a). Consequently, it has become rather widely accepted that non-GIA processes should be integral to explaining the observed SPM [10, 11, 12]. Quantifying the relative importance of such contributions, however, has been hampered by the relatively poorly treated statistics of the GIA predictions of SPM.

Here we employ a GIA model 13] that operates on a robust Bayesian statistical framework (Supplementary Methods Section 1). Our model has a radially

45 symmetric solid Earth structure, with one lithosphere and two mantle layers, that may be sufficient to evaluate statistics of low-degree gravity coefficient change and resulting polar motion. We assemble a global distribution of paleo relative sea-level (RSL) data from 11,451 sites and Global Positioning System (GPS) data from 459 stations. We have carefully selected these data sets and 
corrected, when applicable, for contemporary ice loss to ensure that these are minimally contaminated by non-GIA signals. We build a cost function, to be minimized, by ingesting all of these global data sets into our Bayesian framework, with a proper accounting of data uncertainty and redundancy, in order to explore parameter space related to solid Earth structure and deglaciation history simultaneously. One approach often taken is to use the observed polar motion as a necessary constraint on lower mantle viscosity structure [14. Here we do not provide such rotational constraints because our goal is to cleanly quantify GIA-driven SPM, given that other drivers are present. Our Bayesian analysis therefore unburdens the GIA model from seeking full reconciliation of observed SPM. What emerges is the probability distribution function - based on a set of 128,000 model realizations - for the present rate of GIA-driven SPM (Figure 1b).

It is important to appreciate the sensitivity of the predicted SPM with respect to the GIA model parameters. Here we explore a total of eight parameters

65 (Figure 2), three of which are related to solid Earth structure and five to the relative ice volumes involved in deglaciation since the Last Glacial Maximum. The glaciation parameters basically scale the ice volume of the reference ice models [15. 16] in five different regions independently. Figure 2 suggests the following two key points: (1) as noted in past studies [2, 6, 11, 12, SPM predictions are 70 most sensitive to lower mantle viscosity; and (2) as depicted by the clustering of "likely" models, all of the model parameters are fairly well resolved by the constraining data sets. Our preferred models have upper and lower mantle viscosities in the respective ranges of $(3.6-10) \times 10^{20} \mathrm{~Pa} \mathrm{~s}$ and $(7-73) \times 10^{21}$ $\mathrm{Pa}$ s. These are in agreement with the average profiles of many GIA models 75 [10, 15, 16], including those that account for rotational constraints [12, and with a number of mantle convection models, some of which are considered later in this study [17. Our estimates are also consistent with viscosities inferred, for example, from Satellite Laser Ranging measurements of low-degree gravity field [18, mineral physics [19], and sinking rate of subducted lithosphere [20].

We estimate that GIA presently causes the Earth's spin axis to drift along 
$79.4^{\circ} \pm 2.9^{\circ}$ west longitude at a speed of $3.5 \pm 1.9 \mathrm{~cm} /$ year, accounting for only $33 \pm 18 \%$ of the observed SPM amplitude. Predicted direction, as in many GIA models [3, 11, aligns well with the observation with a difference of only $5.2^{\circ} \pm$ $5.5^{\circ}$. Our estimate of SPM amplitude is within the plausible range of estimates recently computed using the improved theory of ice age Earth's rotation [7], 10, 11, 12]. For example, a theoretical model accounting for a restoring torque associated with the triaxiality of the Earth's inertia tensor [10] predicts that GIA cannot explain more than $70 \%$ of the observed SPM. Whereas another model, constrained by secular rate of degree- 2 zonal gravity coefficient, having a low viscosity D" layer above the core-mantle boundary [12] predicts an even smaller contribution from GIA $(\sim 45 \%)$. It is important to recognize that our estimates are independently derived using a Bayesian approach to assimilate a global distribution of $\sim 12,000 \mathrm{RSL} / \mathrm{GPS}$ data, and hence represent the statistically most robust estimates available to date.

\section{Environmental processes}

Considering the recent discovery that the transfer of mass between the continents and oceans may dramatically shift the general drift direction of Earth's spin axis [21, 22, we assess the effect of environmental processes on the 20th century SPM. Climate reconstructions that account for land-ocean mass transport are essential to deciphering the anthropogenic signals in the data record. Consequently, 20th century atmospheric, land hydrology, ocean heat exchange and cryospheric mass balance reconstructions have made significant advancement in the past decade. Each potential contributor to the 20th century GMSL rise is now robustly estimable [23, 24, 25, 26, 27, including those which are by direct human intervention [23, 24. We collect all sources of continental mass changes (Table 1) and solve for the associated ocean mass redistribution, constrained by the mass conservation principle, on an elastically compressible, self-gravitating, rotating Earth [28] to develop a complete global model of Earth's surface mass transport and robustly quantify the associated SPM (Supplementary Methods 
Section 2).

The response is considered for global glaciers that include 18 of the 19 regions of the Randolph Glacier Inventory (RGI). This part of the cryosphere has been modeled using the 20th century climate data [26] and been shown to be consistent with glaciological observations (e.g., surface mass balance, glacier volume change). The 20th century evolution of the Greenland Ice Sheet, with high-resolution maps of ice thinning, has been constrained by combining aerial imagery and measurements form airborne and satellite altimetry [25]. While the evolution of Antarctic Ice Sheet and its peripheral glaciers - the 19th RGI region - are more uncertain, several lines of evidence suggest that much of the Antarctic Peninsula has been losing mass since the Little Ice Age maximum [29, 30], the West Antarctica (and the Amundsen Sea Sector especially) has been thinning at least since the 1930s [27, and East Antarctica has remained mostly stable, with its outlet glaciers fluctuating on interdecadal timescales [31] and its surface mass balance showing no apparent trends [32], at least during the latter half of the 20th century.

In addition to these cryospheric sources, the 20th century rise in GMSL has also been affected by sustained changes in terrestrial water storage, primarily related to ground water depletion and artificial reservoir water impoundment. Model-based, observationally-constrained global estimates of ground water depletion 24] show accelerated mass loss in the continental US and the Indian subcontinent throughout the 20th century. Total reservoir water impoundment - based on the storage capacity and seepage potential of the world's 29,000 large dams - is estimated to be significant enough to lower the 20th century GMSL rise by about $29 \mathrm{~mm}[23$. Other anthropogenic terrestrial sources (e.g., deforestation, wetland and endorheic basin storage loss) are nominal, and changes in natural storage (e.g., soil moisture, permafrost, snow) are assumed to be limited to interannual to decadal variability [33]. The combined cryospheric and hydrologic contributions falls short of the more likely rate of the 20th century GMSL: 1 to $2 \mathrm{~mm} /$ year (Table 1 ). We consider the residual as a steric sea-level change 40 that may also redistribute the ocean mass internally, inducing a non-negligible 
SPM [34.

Our estimates of SPM driven by all sea-level sources mentioned above, and accompanying ocean mass redistributions, show greatly varying amplitudes and directions (Figure 3). High-mountain Asian glaciers, together with those in the Southern Andes, play a stronger role than do the combined effects of Alaskan and peripheral Greenland glaciers in controlling the net SPM associated with global glaciers and ice caps. We assume that atmospheric excitations (due to winds and surface pressure variations) are negligible on secular timescales [1]. Our estimate of the sum of all environmental processes is that they drive a net SPM along $19.2^{\circ} \pm 16.1^{\circ}$ east longitude at a speed of $4.3 \pm 1.0 \mathrm{~cm} /$ year averaged over the 20th century (Table 1). This environmental polar motion during 20032015 points slightly eastwards (along $27.7^{\circ}$ east longitude) but at a much faster rate $(14.2 \mathrm{~cm} /$ year) [22], revealing a global geodetic imprint of accelerated mass loss from Greenland, West Antarctica, and ground water. We emphasize the importance of global mass conservation (i.e., inclusion of induced ocean mass redistribution due to self-attraction and loading), as it accounts for about $27 \%$ of the environmental SPM amplitude, with minimal effects on the predicted direction.

\section{Residual motion and mantle convection}

Coseismic and interseismic deformations tend to operate in opposing directions and the sum yields a small excitation (on the order of $0.8 \mathrm{~cm} /$ year along $117^{\circ}$ west longitude) during the 20th century [35. The summed GIA, environmental and seismogenic signals - when subtracted from the observed SPM - yields a residual motion that points along $101.1^{\circ} \pm 9.7^{\circ}$ west longitude at a speed of $7.4 \pm 2.4 \mathrm{~cm} /$ year (Figure 4). Finally, to explain this large residual, we are compelled to turn to inertia changes owing to mantle convection.

Over geological timescales, global plate motions are reconstructed from geomagnetic reversals recorded at mid-ocean ridges and hotspot tracks. Combining these with paleomagnetic data from the continents, the "true" position of the 
This "True Polar Wander" (TPW) can be modeled geodynamically, because the spin axis follows the maximum inertia axis, which can be determined from the degree-2 geoid. Although advanced geodynamical models now incorporate the delayed viscous adjustment of the rotational bulge [37, 17], we mostly disregard this effect here. This is because the adopted viscosities and the inferred rates of TPW are such that the spin axis should follow very closely the maximum inertia axis associated with mantle convection 38, as confirmed by the one case considered in our calculations where the effect of rotational bulge adjustment is investigated. The evolving density anomalies in geodynamical models, which give rise to geoid changes, can be inferred from global subduction histories and the sinking rate of slabs [39, 17], or from density and flow models [40, 38] based on seismic tomographic observations [41].

Here, we assemble "present" rates of TPW - termed mantle convection driven SPM - computed by contemporary geodynamical models without an 3). We retrieve 94 modeled rates of TPW, each for the last 2 and 10 million years, from a suite of slab subduction driven forward models that were based on plate motions and aimed at reconstructing 3-D seismic structure and the geoid [17. These are computed assuming a realistic viscosity structure [19], which is compatible with - though not precisely the same as - the inferred viscosity profile in our GIA Bayesian inversions [13]. We also perform a total of 95 new computations using a backward-advection approach 38] that ingests a suite of seismic tomographic models and viscosity structures toward reconstructing the past plate motions. We vary a set of key parameters (e.g., tomographic models, viscosity structure, factors that translate seismic velocities into density anomalies) and adjust either the degree- 2 geoid coefficients to match the actual present-day coefficients or shift the TPW curve itself to match the presentday pole position. We retrieve the predictions of TPW rate for the last 0.01 or 1 million years and find that these solutions give broadly similar results as 
in speed. Note that TPW rates are originally computed in "mantle reference frame", which are later projected onto the "mean lithospheric reference frame" in which both the observed and modeled SPM are evaluated.

Comparing the convection predictions to our SPM residual (Figure 4) suggests that the average model direction is, in fact, statistically indistinguishable from the SPM residual direction, with a difference of only $2.7^{\circ} \pm 14.8^{\circ}$. Some predictions fully reconcile the observed SPM, although these exceed TPW rates constrained by paleomagnetics [42]. We speculate that incorporating the residual SPM, either as a constraint or a diagnostic, in future mantle convection models might offer fruitful new insights to the geodynamic processes.

\section{Concluding remarks}

We put forth a new multidisciplinary framework to explain the 20th century polar motion. For the first time, a suite of environmental (e.g., cryospheric, hydrologic, oceanic) and solid Earth geophysical (e.g., GIA, mantle convection, ${ }_{215}$ seismological) processes are all brought together in an effort to explain SPM. These processes operate on a wide range of timescales, but each transports mass and perturbs the Earth's inertia tensor in an entirely quantifiable way. Our account of GIA statistics is comprehensive and the environmental processes are brought together using state-of-the-art models and data sets. Any individual mechanism only provides a partial source for the reconstruction of the SPM observations. This leads us to hypothesize that mantle convection is one key process to consider in greater detail. Indeed, our mantle convective simulations - not designed to comply with any requirements for treating 20th century polar motion - when combined with GIA, environmental and seismogenic SPM, tend to strongly reduce the misfit to the observations.

It is an open question as to how future modeling will progress to achieve a more robust reconciliation. Given the inherent uncertainties associated with seismic tomography, modeling slab density and advective history, and the general non-uniqueness in the interpretation of the low-degree geoid, we speculate 

components of internal mass transport treated in a rigorously consistent manner.

\section{References}

[1] R. Gross, Earth rotation variations - Long period, in: J. Herring (Ed.), Treaties on Geophysics: Physical Geodesy, Vol. 11, Elsevier, Amsterdam, 2007, pp. 215-261.

[2] R. Sabadini, W. Peltier, Pleistocene deglaciation and the Earth's rotation: implication for mantle viscosity, Geophys. J. R. Astr. Soc. 66 (1981) 553-

240

[3] P. Wu, W. Peltier, Pleistocene deglaciation and the Earth's rotation: a new analysis, Geophys. J. R. Astr. Soc. 76 (1984) 753-791.

[4] G. Spada, Y. Ricard, R. Sabadini, Excitation of true polar wander by subduction, Nature 360 (1992) 452-454.

245 [5] Y. Ricard, G. Spada, R. Sabadini, Polar wandering on a dynamic Earth, Geophys. J. Int. 113 (1993) 284-298.

[6] L. Vermeersen, A. Fournier, R. Sabadini, Changes in rotation induced by pleistocene ice masses with stratified analytical earth models, J. Geophys. Res. 102 (1997) 2156-2202.

[7] J. Mitrovica, J. Wahr, I. Matsuyama, A. Paulson, The rotational stability of an ice-age earth, Geophys. J. Int. 161 (2005) 491-506.

[8] W. Munk, Twentieth century sea level: An enigma, PNAS 99 (2002) 65506555 .

[9] J. Mitrovica, C. Hay, E. Morrow, R. Kopp, M. Dumberry, S. Stanley, Reconciling past changes in Earth's rotation with 20th century global sea-level rise: Resolving Munk's enigma, Sci. Adv. 1 (2015) e1500679. 
[10] G. Cambiotti, Y. Ricard, R. Sabadini, Ice age True Polar Wander in a compressible and non-hydrostatic Earth, Geophys. J. Int. 183 (2010) 12481264.

[11] J. Mitrovica, J. Wahr, Ice age Earth rotation, Annu. Rev. Earth Planet. Sci. 39 (2011) 577-616.

[12] M. Nakada, J. Okuno, K. Lambeck, A. Purcell, Viscosity structure of Earth's mantle inferred from rotational variations due to GIA process and recent melting events, Geophys. J. Int. 202 (2015) 976-992.

[13] L. Caron, E. Ivins, E. Larour, S. Adhikari, J. Nilsson, G. Blewitt, GIA model statistics for GRACE hydrology, cryosphere and ocean science, Geophys. Res. Lett. 45 (2018) 2203-2212.

[14] G. Kaufmann, K. Lambeck, Glacial isostatic adjustment and the radial viscosity profile from inverse modeling, J. Geophys. Res. 107 (2002) B112280.

[15] K. Lambeck, A. Purcell, J. Zhao, N. Svensson, The Scandinavian ice sheet: from MIS 4 to the end of the Last Glacial Maximum, Boreas 39 (2010) 410-435.

[16] K. Lambeck, H. Rouby, A. Purcell, Y. Sun, M. Sambridge, Sea level and global ice volumes from the Last Glacial Maximum to the Holocene, PNAS 11 (2014) 15296-15303.

[17] B. Steinberger, M.-L. Seidel, T. Torsvik, Limited true polar wander as evidence that Earth's nonhydrostatic shape is persistently triaxial, Geophys. Res. Lett. 44 (2017) 827-834.

[18] N. Tosi, R. Sabadini, A. Marotta, L. Vermeersen, Simultaneous inversion for the Earth's mantle viscosity and ice mass imbalance in Antarctica and Greenland, J. Geophys. Res. 110 (2005) B07402. 
[19] B. Steinberger, A. Calderwood, Models of large-scale viscous flow in the Earth's mantle with constraints from mineral physics and surface observations, Geophys. J. Int. 167 (2006) 1461-1481.

[20] H. Cizkova, A. P. van den Berg, W. Spakman, C. Matyska, The viscosity of Earth's lower mantle inferred from sinking speed of subducted lithosphere, Phys. Earth Planet. Inter. 200-201 (2012) 56-62.

[21] J. Chen, C. Wilson, J. Ries, B. Tapley, Rapid ice melting drives Earth's pole to the east, Geophys. Res. Lett. 40 (2013) 2625-2630.

[22] S. Adhikari, E. Ivins, Climate-driven polar motion: 2003-2015, Sci. Adv. 2 (2016) e1501693.

[23] B. Chao, Y. Wu., Y. Li, Impact of artificial reservoir water impoundment on global sea level, Science 320 (2008) 212-214.

[24] Y. Wada, L. van Beek, F. Weiland, B. Chao, Y. Wu, M. Bierkens, Past and future contribution of global groundwater depletion to sea-level rise, Geophys. Res. Lett. 39 (2012) L09402.

[25] K. Kjeldsen, N. Korsgaard, A. Bjork, S. Khan, J. Box, S. Funder, N. Larsen, J. Bamber, W. Colgan, M. van den Broeke, M.-L. Siggaard-Andersen, C. Nuth, A. Schomacker, C. Andresen, E. Willerslev, K. Kjaer, Spatial and temporal distribution of mass loss from the Greenland Ice Sheet since AD 1900, Nature 528 (2015) 396-400.

[26] B. Marzeion, P. Laclercq, J. Cogley, A. Jarosch, Brief Communication: Global reconstructions of glacier mass change during the 20th century are consistent, The Cryosphere 9 (2015) 2399-2404.

[27] J. Smith, T. Andersen, M. Shortt, A. Gaffney, M. Truffer, T. Stanton, R. Bindschadler, P. Dutrieux, A. Jenkins, C.-D. Hillenbrand, W. Ehrmann, H. Corr, N. Farley, S. Crowhurst, D. Vaughan, Sub-ice-shelf sediments record history of twentieth-century retreat of Pine Island Glacier, Nature 541 (2017) 77-80. 
[28] S. Adhikari, E. Ivins, E. Larour, ISSM-SESAW v1.0: Mesh-based computation of gravitationally consistent sea level and geodetic signatures caused by cryosphere and climate driven mass change, Geosci. Model Dev. 9 (2016) 1087-1109.

[29] A. Christ, M. Talaia-Murray, N. Elking, E. Domack, A. Leventer, C. Lavoie, S. Brachfeld, K.-C. Yoo, R. Gilbert, S.-M. Jeong, S. Petrushak, J. Wellner, the LARISSA Group, Late Holocene glacial advance and ice shelf growth in Barilari Bay, Graham Land, west Antarctic Peninsula, Vol. 31035, Geological Society of America Bulletin, 2014.

320 [30] A. Cook, A. Fox, D. Vaughan, J. Ferrigno, Retreating glacier fronts on the Antarctic Peninsula over the past half-century, Science 308 (2005) 541-544.

[31] B. Miles, C. Stokes, S. Jamieson, Pan-ice-sheet glacier terminus change in East Antarctica reveals sensitivity of Wilkes Land to sea-ice changes, Sci. Adv. 2 (2016) e1501350.

${ }_{325}$ [32] A. Monaghan, D. H. Bromwich, R. L. Fogt, S.-H. Wang, P. A. Mayewski, D. A. Dixon, A. Ekaykin, M. Frezzotti, I. Goodwin, E. Isaksson, S. D. Kaspari, V. I. Morgan, H. Oerter, T. D. Van Ommen, C. J. Van der Veen, J. Wen, Insignificant change in Antarctic snowfall since the International Geophysical Year, Science 313 (2006) 827-831.

330 [33] J. Reager, A. Gardener, J. Famiglietti, D. Wiese, A. Eicker, M. Lo, A decade of sea level rise slowed by climate-driven hydrology, Science 351 (2016) 699-703.

[34] F. Landerer, J. Jungclaus, J. Marotzke, Long-term polar motion excited by ocean thermal expansion, Geophys. Res. Lett. 36 (2009) L17603.

335 [35] G. Cambiotti, X. Wang, R. Sabadini, D. Yuen, Residual polar motion caused by coseismic and interseismic deformations from 1900 to present, Geophys. J. Int. 205 (2016) 1165-1179. 
[36] J. Besse, V. Courtillot, Apparent and true polar wander and the geometry of the geomagnetic field over the last 200 Myr, J. Geophys. Res. 107 (2002) N11-2300.

[37] G. Cambiotti, Y. Ricard, R. Sabadini, New insights into mantle convection true polar wander and rotational bulge readjustment, Earth Planet Sci. Lett. 310 (2011) 538-543.

[38] B. Steinberger, R. O'Connell, Changes of the Earth's rotation axis owing to advection of mantle density heterogeneities, Nature 387 (1997) 169-173.

[39] M. Domeier, P. Doubrovine, T. Torsvik, W. Spakman, A. Bull, Global correlation of lower mantle structure and past subduction, Geophys. Res. Lett. 43 (2016) 4945-4953.

[40] Y. Ricard, M. R. amd C. Lithgow-Bertelloni, Y. Le Stunff, A geodynamic model of mantle density heterogeneity, J. Geophys. Res. 98(B12) (1993) 21895-21909.

[41] S. French, B. Romanowicz, Whole-mantle radially anisotropic shear velocity structure from spectral-element waveform tomography, Geophys. Res. Lett. 199 (2014) 1303-1327.

[42] P. Doubrovine, B. Steinberger, T. Torsvik, Absolute plate motions in a reference frame defined by moving hot spots in the Pacific, Atlantic, and Indian oceans, J. Geophys. Res. 117 (2012) B09101.

[43] W. Peltier, Global glacial isostasy and the surface of the ice-age Earth: The ICE-5G (VM2) model and GRACE, Annu. Rev. Earth Planet. Sci. 32 (2004) 111-149.

\section{Acknowledgements}

This research was carried out at the Jet Propulsion Laboratory (JPL), California Institute of Technology, under a contract with National Aeronautics and 
Space Administration (NASA), and was primarily funded through the JPL Re-

Conversations with Felix W. Landerer are acknowledged.

\section{Author contributions}

S.A. and E.R.I. conceived the research and wrote the first draft of the manuscript. L.C. and B.S. conducted GIA and mantle convection simulations, respectively, and contributed to the interpretation of the results, as well as to the writing of the manuscript. S.A. led the rest of the calculations, with help of all other authors. All authors reviewed and approved the final draft of the manuscript. 
Table 1: Sources of the 20th century GMSL rise and excitation of SPM. Components of the SPM vector are projected along the central Greenwich meridian, $\dot{m}_{1}$, and the $90^{\circ}$ east longitude, $\dot{m}_{2}$. See Supplementary Methods Section 2 for a description on how we assemble the GMSL sources and compute SPM.

\begin{tabular}{ccccc}
\hline Source & $\begin{array}{c}\text { GMSL rate } \\
{\left[\mathrm{mm} \mathrm{yr}^{-1}\right]}\end{array}$ & $\begin{array}{c}\text { SPM rate } \dot{m}_{1} \\
{\left[\mathrm{~cm} \mathrm{yr}^{-1}\right]}\end{array}$ & $\begin{array}{c}\text { SPM rate } \dot{m}_{2} \\
{\left[\mathrm{~cm} \mathrm{yr}^{-1}\right]}\end{array}$ & References \\
\hline Global glaciers & $0.60 \pm 0.04$ & $1.46 \pm 0.33$ & $2.19 \pm 0.35$ & {$[26]$} \\
Greenland Ice Sheet & $0.21 \pm 0.04$ & $2.47 \pm 0.92$ & $-2.03 \pm 0.68$ & {$[25]$} \\
Antarctic Ice Sheet & $0.05 \pm 0.04$ & $-0.16 \pm 0.29$ & $0.69 \pm 0.72$ & 30, 32, 29, 31, 27] \\
Groundwater & $0.17 \pm 0.04$ & $0.77 \pm 0.32$ & $1.50 \pm 0.62$ & {$[24]$} \\
Dams/reservoirs & -0.29 & -0.14 & -0.78 & {$[23]$} \\
Steric & {$[0.26,1.26]$} & $-0.37 \pm 0.28$ & $-0.15 \pm 0.12$ & {$[34]$} \\
\hline Total & {$[1.0,2.0]$} & $4.03 \pm 1.10$ & $1.42 \pm 1.23$ & \\
\hline
\end{tabular}



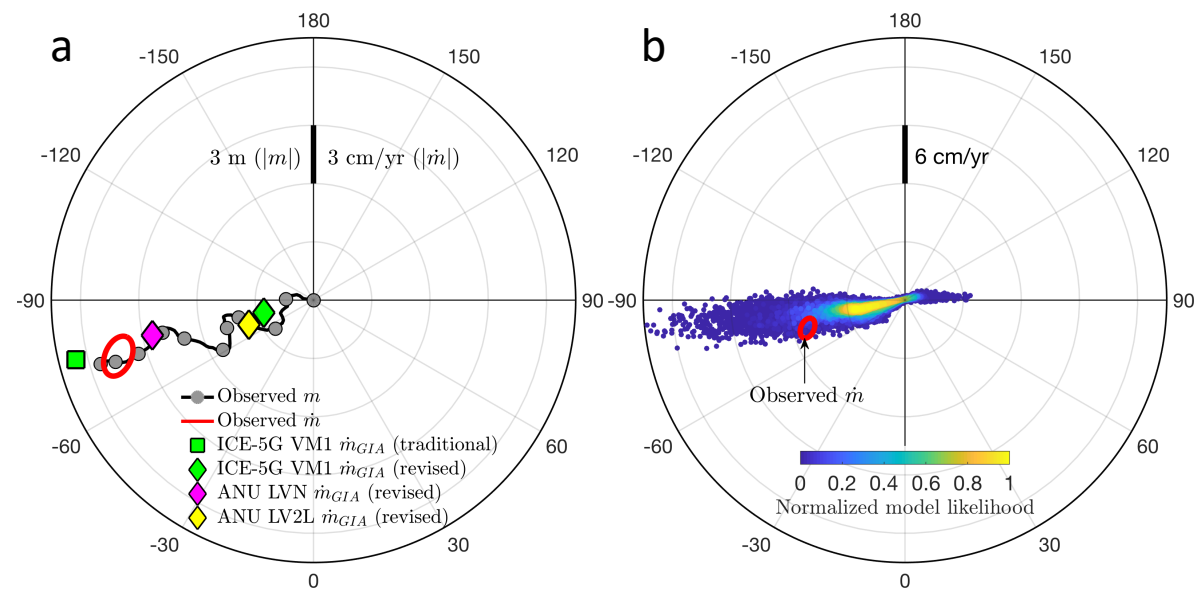

Figure 1: GIA and the 20th century SPM. (a) Observed and modeled rates of SPM, $\dot{m}$. All model predictions, $\dot{m}_{G I A}$, are solely due to GIA processes. The predictions differ in assumed deglaciation history (ICE-5G [4] or ANU 15, 16]) and in mantle viscosity profile (VM1 [43, LVN or LV2L [12]). The restoring torque effect is highlighted by showing predictions (green markers) computed by using the "traditional" 3 or the "revised" ice age rotational stability theory [7. Even after accounting for the improved rotational stability, some models nonetheless predict significant $\dot{m}_{G I A}$ (magenta diamond; see also 9]). Inclusion of a low viscosity D" layer, however, dampens the amplitude (yellow diamond). This non-uniqueness in $\dot{m}_{G I A}$ solutions motivates the statistically robust new Bayesian assessment (see panel b). Observed mean pole positions, $m(t)$, relative to 1900 is shown (data courtesy of International Earth Rotation and Reference Systems Service: https://www.iers.org/) to note that the spin axis does not drift in a precisely linear path. A low pass filter having a 6-year window allows interannual signals to be seen. Gray circles represent the mean annual positions at 10-year time intervals. The same scale bar with differing metric is used for $m$ and $\dot{m}$. (b) Our predicted $\dot{m}_{G I A}$ for 128,000 models. The color scale represents the likelihood of a given model to explain the global RSL/GPS data. Our predictions generally align with the observed $\dot{m}$, with many (less-likely) models fully reconciling the observation. The Bayesian statistics suggest that GIA accounts for only $33 \pm 18 \%$ of the observed SPM amplitude. Note that we have different scales on panels a and $\mathrm{b}$. 

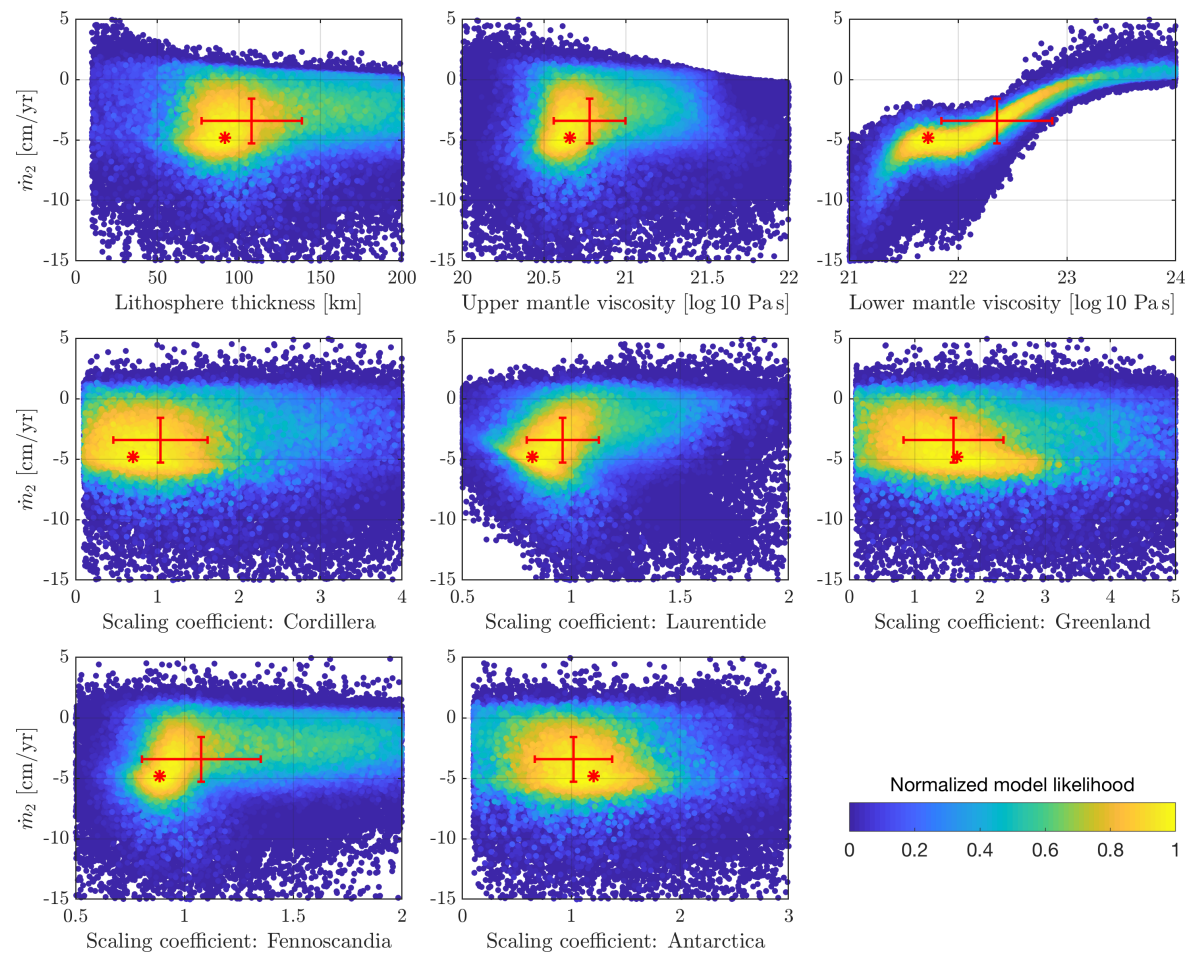

Figure 2: Sensitivity of predicted SPM with respect to GIA model parameters. To demonstrate the sensitivities in both amplitude and direction, results are shown for the SPM rate vector projected along the $90^{\circ}$ east longitude, denoted here by $\dot{m}_{2}$. Likelihood probability distributions - normalized by the best-fit model probability - are projected in 2-D spaces formed by $\dot{m}_{2}$ and each of 8 model parameters. Both solid Earth parameters (upper panel) and relative ice volume involved in deglaciation (middle and lower panels) are considered. Stronger sensitivities are evident for lower mantle viscosity. Red stars denote the "best-fit" model solution. Expected values and 1- $\sigma$ uncertainties for $\dot{m}_{2}$ and the model parameters are shown by vertical and horizontal error bars, respectively. Note that models with larger probability are plotted on top of those with lower probability. 

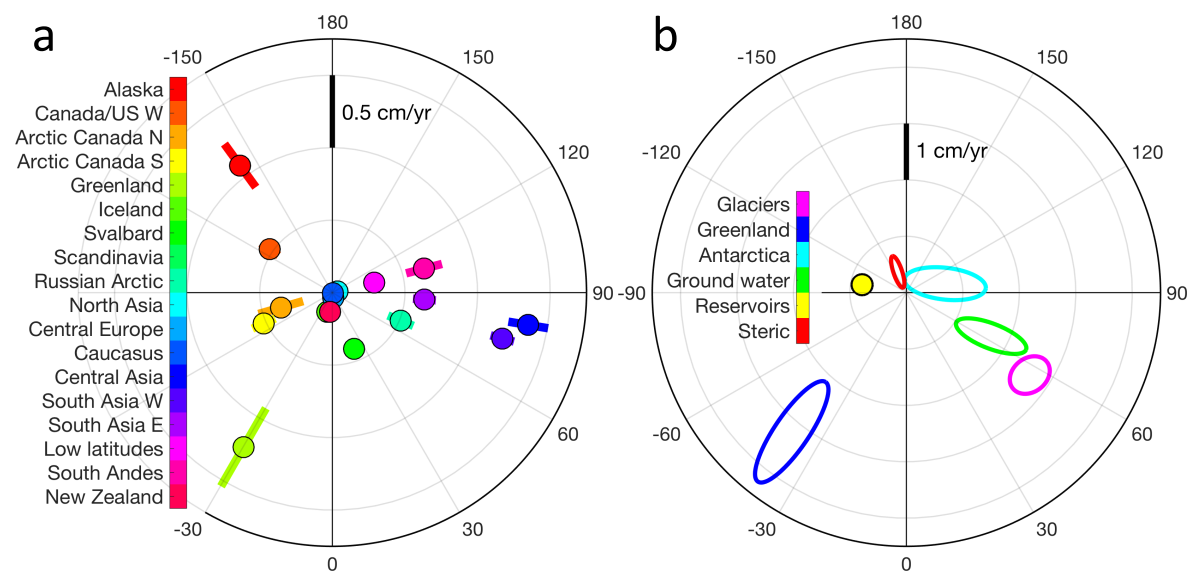

Figure 3: Environmental excitations of the 20th century SPM. (a) SPM driven by melting of regional glaciers and ice caps and accompanying ocean mass redistributions. For assumed uniform melting of individual RGI regions, there is no uncertainty for the predicted direction. Therefore, uncertainty estimates are provided only for the SPM amplitudes. (b) Combined SPM due to melting of these 18 RGI glaciated regions (pink ellipse), along with those induced by other cryospheric, hydrologic, and oceanic sources of 20th century GMSL rise (see Table 1). The ellipses represent the uncertainties. Note that we have different scales on panels a and $b$. 

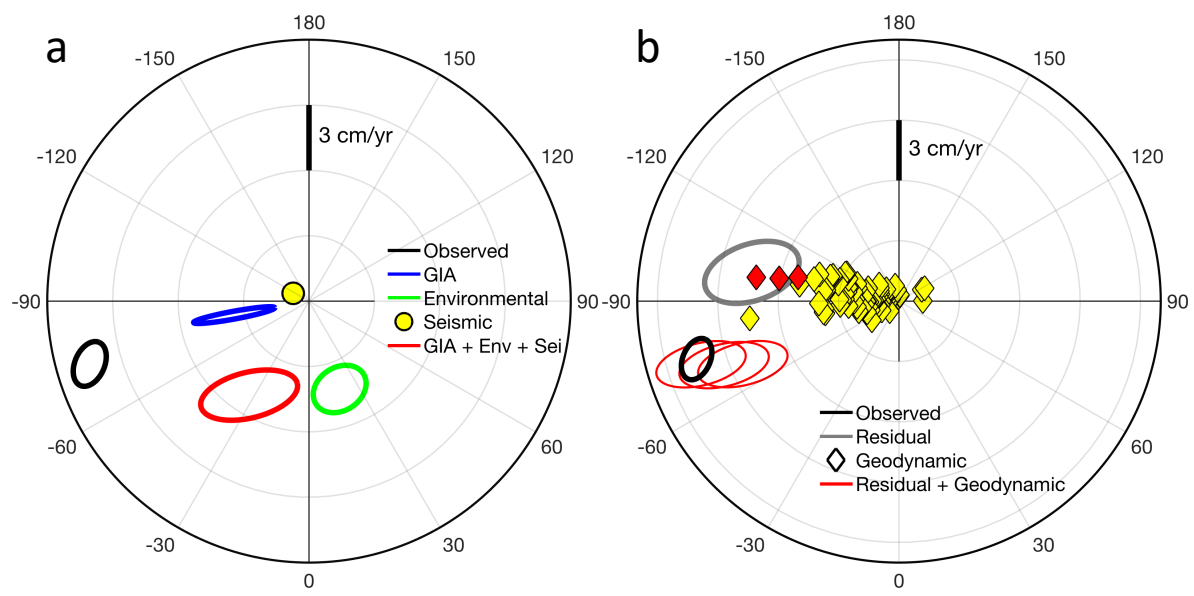

Figure 4: Budget analysis of the observed SPM. (a) We provide robust statistics of SPM induced by GIA and environmental processes. The summed signal (red ellipse), including seismic SPM, is far from reconciling the observation (black ellipse). (b) To explain large residual (gray ellipse), we assemble a total of 283 mantle convection model predictions. A full description of all of these models is given in Supplementary Methods Section 3. Here, for simplicity, we only consider 82 representative solutions (diamonds) that were retrieved for the last 0.01 million years from backward advection models. While predicted amplitudes have considerable scatter, the general direction of mantle convection driven SPM aligns with that of the residual motion. In fact, some of these predictions (e.g., red diamonds) when combined with other excitation sources tend to reconcile the observation (see black vs. red ellipses), but the associated amplitudes exceed the TPW rates constrained by paleomagnetics 42 . 